\title{
Epidemiology and Clinical Characteristics of Covid-19 Mortality in Jos, Nigeria
}

\author{
Gomerep SS ${ }^{1^{*}}$, McHenry $\mathrm{SI}^{2}$, Kumbak $\mathrm{FD}^{3}$, Galam $\mathrm{NZ}^{4}$, Ogwuche $\mathrm{JE}^{2}$, Bartekwa $\mathrm{JW}^{2}$, \\ Bako IB $^{2}$, Shehu NY2 $\mathrm{N}^{2}$, Isa SE${ }^{1}$. \\ ${ }^{1}$ Department of Medicine, University of Jos, Jos Nigeria \\ ${ }^{2}$ Department of Medicine, Jos University Teaching Hospital, Jos Nigeria \\ ${ }^{3}$ Department of Community Medicine, Jos University Teaching Hospital, Jos Nigeria \\ ${ }^{4}$ Department of Physiology, University of Jos, Nigeria \\ *Corresponding Author: Gomerep S, S. simjoma04@gmail.com.+2348033568566
}

\begin{abstract}
Background: Coronavirus Disease 2019 mortality figures in Africa are comparatively lower than the figures in Europe, Asia and the Americas. Many reasons have been adduced for the differences which include the younger population in Africa. We therefore out set to describe the epidemiology and clinical features among in-hospital mortalities in our setting.
\end{abstract}

Methods: This was a retrospective study of all mortalities due to confirmed COVID-19 at the Jos University Teaching Hospital from April 2020 to April 2021. Descriptive statistics were used to present results and Chi Square analysis used to determine the association between morbidities, sex and ventilator use. A p-value of $<0.05$ was considered statistically significant.

Results: A total of 80 mortalities were recorded over the 12 month period. Sixty one (76.0\%) were males. The mean age of the study population was $61 \pm 15$ years and twenty nine (36.3\%) were aged $\leq 59$ years. The median duration of hospitalization for in-hospital mortality was 4days (IQR 135). Fever; 61(76.3\%), cough; 59(73.8\%) and dyspnea; $56(70.0 \%)$ were the commonest presenting symptoms and 35(43.8\%) had the three symptoms. Hypertension; 48(60.0\%) and type 2 diabetes mellitus; $36(45.0 \%)$ were the commonest co-morbidities in the patients, and $31(38.8 \%)$ had at least two co-morbidities. Eighteen (22.5\%) had both hypertension and diabetes mellitus. Hypertension and having two or more co-morbidities where associated with requirement for ventilator support $(P<0.013$ and $P<0.001$ respectively).

Conclusion: Fever, cough, dyspnea and two or more co-morbidities are common among COVID-19 mortalities in Jos. Efforts to quickly identify such patients and manage comorbidities are needed to reduce mortality.

Keywords: COVID-19 mortality; Comorbidity; Clinical features;

Introduction

Since late 2019 when the earliest cases of

COVID-19 were detected in Wuhan
China, the disease has gone from an epidemic to a pandemic as declared by the World Health Organization (WHO) on the 
$11^{\text {th }}$ of March $2020^{1}$. Following the declaration, there has been a myriad of actions reactions and inactions ${ }^{2}$, and the death toll has continued to rise globally with over 3.8 million deaths out of the over 179 million confirmed cases as at June 26, 2021. ${ }^{3}$ There have been differences in mortality rates across the globe with countries like the USA, Brazil and recently India being the worst hit. Surprisingly, such high mortality has not been noticed in most of sub-Saharan Africa. ${ }^{4}$

Several factors have been shown to influence mortality in COVID-19 patients. These include obesity, poorly controlled glycaemia in diabetes patients, hypertension, older age and other forms of immunosuppressing states like Chronic Kidney Disease (CKD), and use of anticancer drugs. ${ }^{5,6,7}$ In a study estimating risk factors of COVID-19 mortality, age $\geq 60$ years was the highest risk factor for mortality with more than 18 times increased risk and males being more likely to die from the disease. They also found cardiovascular diseases had more than 12 times increased risk and chronic respiratory diseases more than 7 times increased risk. ${ }^{8}$

In Western Cape province, South Africa, HIV and tuberculosis infection were risk factors for increased mortality in their cohort. ${ }^{9}$ To understand the variation in mortality in Africa compared to Europe and the Americas, some factors have been postulated to explain the differences; the fewer reported cases, and therefore, mortality in Africa was being attributed to low rate of testing for the disease in the continent as against higher testing rates in the western world and Asia, the younger population, higher vitamin $\mathrm{D}$ levels in Africans as a result of exposure to sunlight, cross-immunity from other viruses including coronaviruses, and lessons learnt from other infectious diseases such as HIV and Ebola may have contributed to the lower mortality rate. ${ }^{10}$

In Nigeria, the story has not been different. Since the $27^{\text {th }}$ of February 2020 when Nigeria recorded its first case, ${ }^{11}$ about 2,119 individuals have died out of over 167,430 confirmed cases as at June 26, 2021. The first case of COVID 19 was confirmed in Jos, Plateau State on the $23^{\text {rd }}$ of April 2021 which heralded the beginning of the epidemic in the State. Plateau State has ranked among the top 4 states with the highest number of cases in the country. ${ }^{12}$ In Nigeria, states' GDP per capita and population density appeared to be moderate risk factors for COVID-19 case mortality per million population whereas BCG vaccination coverage and HIV prevalence appeared to afford some protection. ${ }^{13}$ In another study in Lagos, Nigeria, COVID 19 mortality and severe symptoms were significantly higher in hypertensive patients and having additional co-morbidities lowers survival. Severe symptoms and mortality were significantly higher among the hypertensive and survival rates were significantly lowered by the presence of an additional comorbidity to $50 \%$ from $91 \%$ for those with hypertension alone and from $98 \%$ for all other patients. ${ }^{14}$

The epidemiological and clinical characteristics of patients who died of COVID-19 in terms of the transmission, detailed clinical course of illness, risk factors for mortality and its treatment are still being studied globally but at a slower pace in resource limited settings. Hence this study was aimed at describing the epidemiology and clinical characteristics of cases of COVID-19 mortality. This will help in quick identification of patients who are high risk for mortality and also provide baseline data for Low and Middle Income (LMICs). 


\begin{abstract}
Methods
Study setting

This was a retrospective study of all the cases of COVID-19 in-hospital mortality from April 2020 to April 2021 at the Jos University teaching Hospital (JUTH), a 500 bed capacity Federal Government tertiary hospital. The hospital has a 32 bed Isolation capacity and a 4 bed intensive Care capacity in the isolation unit. Jos University Teaching Hospital receives referrals from surrounding communities and neighboring States of Nasarawa, Taraba, Benue, Kaduna and Bauchi.
\end{abstract}

\section{Data collection}

All cases were confirmed by positive Polymerase Chain Reaction (PCR) test for SARS-CoV-2 on respiratory samples collected by nasopharyngeal and oropharyngeal swab and analyzed at the Nigeria Centre for Disease Control (NCDC) approved laboratories in Jos or the National Reference Laboratory at Abuja. Case record notes of all the mortalities during the period were used to extract relevant socio-demographic and clinical data which included age, sex, occupation, length of stay on admission, presenting symptoms, co-morbidities, date of demise and ventilator use. The data was then entered into an excel sheet and cleaned.

\section{Data analysis}

Quantitative variables such as age of the patients and Oxygen saturation ( $\mathrm{SpO} 2)$ were expressed as mean \pm Standard Deviation (SD), and duration on admission expressed as median days with Interquartile Range (IQR). Qualitative variables such as sex and occupation, symptoms, ventilator use were presented using frequency tables, and expressed as percentages and Chi-square test was used to determine the associations between sex, comorbidity and ventilator use.
Stata software version 13.0 was used for analysis and a p-value of $<0.05$ was considered statistically significant ${ }^{15}$.

\section{Ethical consideration}

Institutional Research Ethics committee of the Jos University Teaching Hospital granted ethical approval for this study $r$ e $f \quad e \quad r \quad e \quad n \quad c e$ JUTH/DCS/IREC/127/XXXI/2506. Data used for this study was de-identified to ensure anonymity. Informed consent was not required for this study as it depended on previously collected service data and a waiver was issued by the ethics committee.

\section{Results}

A total of 80 mortalities were recorded during the period. Sixty one were males and majority were either retired from service; $20(25.0 \%)$ or civil servants; $23(28.8 \%)$. Seventy $(87.5 \%)$ were from Plateau State and Jos North and Jos South Local Government Areas were the predominant residences $(66.3 \%)$ of patients. The mean age of the patients was $61.0 \pm 15.0$ years. Twenty nine $(36.3 \%)$ were aged $\leq 59$ years (Table 1 ).

The median duration on admission was 4 days (IQR 1-35) and a total of 23(29.1\%) patients spent $\geq 9$ days on admission. Only $29(42.0 \%)$ had achieved oxygen saturation $(\mathrm{SpO} 2)$ within normal limits $(\geq 95 \%)$ anytime during the admission and $74(96.1 \%)$ had the lowest recorded $\mathrm{SpO} 2 \leq$ $89 \%$ at any time during the admission (Table 2). Fever; 61(76.35), cough; 59(73.8\%) and dyspnea; $56(70.0 \%)$ were the commonest presenting symptoms. A combination of the three symptoms occurred in $35(43.8 \%)$ of the patients (Figure 1). A total of $62(77.5 \%)$ had at least one comorbidity. Hypertension; $48(60.0 \%)$ and type 2 diabetes mellitus; $36(45.0 \%)$ were the commonest comorbidities in the patients and 
$31(38.8 \%)$ had at least two co-morbidities (Figure 2). Eighteen (22.5\%) had both hypertension and diabetes mellitus.

Twenty eight $(35.0 \%)$ of the patients had ventilator support (Table 3) and 64(80.0\%) died of respiratory failure (Figure 3 ). The mortality trend showed a double peak within the period at the month of July
2020; 21(26.4\%) and in January 2021; $14(17.5 \%)$. The mortality during the first wave (April to October 2020) was higher; $44(55.0 \%)$ than the second (Figure 4). Hypertension and having double comorbidity were associated with ventilator support $(\mathrm{P}<0.013$ and 0.001 respectively)(Table 3 ).

Table 1: Socio-demographic characteristics of the deceased patients

\begin{tabular}{llll}
\hline Variables & Female & Sex & Total \\
\hline Age group (years) & & & \\
$\leq 59$ & $7(36.8)$ & $22(36.1)$ & 29 \\
$60-64$ & $4(21.1)$ & $16(26.2)$ & 20 \\
$65-69$ & $2(10.5)$ & $7(11.5)$ & 9 \\
$\geq 70$ & $6(31.6)$ & $16(26.2)$ & 22 \\
Total & $19(100)$ & $61(100)$ & 80 \\
& Mean $\pm \mathrm{SD}=61.0 \pm 15.0$, Range $; 15-91$ & \\
Occupation & & & 13 \\
Business & $4(21.0)$ & $9(14.7)$ & 23 \\
Civil servant & $3(15.8)$ & $20(32.7)$ & 2 \\
Clergy & $0(0.0)$ & $2(3.3)$ & 4 \\
Driver & $0(0.0)$ & $4(6.6)$ & 8 \\
Farmer & $0(0.0)$ & $8(13.1)$ & 20 \\
Housewife & $5(26.3)$ & $0(0.0)$ & 5 \\
Retired & $6(31.6)$ & $14(23.0)$ & 80 \\
Others & $1(5.3)$ & $4(6.6)$ & \\
Total & $19(100)$ & $61(100)$ & 70 \\
Rsidence (state) & & & 5 \\
Plateau & $17(89.4)$ & $53(86.9)$ & 2 \\
Benue & $0(0.0)$ & $5(8.3)$ & 2 \\
Kaduna & $1(5.3)$ & $1(1.6)$ & 1 \\
Nasarawa & $1(5.3)$ & $1(1.6)$ & 80 \\
Taraba & $0(0.0)$ & $1(1.6)$ & 61 \\
Total & 19 & & \\
\hline & & 61.0 & \\
\hline
\end{tabular}


Table 2: Clinical Characteristics of the deceased patients

\begin{tabular}{ll}
\hline Variable & Frequency (\%) \\
\hline Days on admission (days) & \\
$\leq 2$ & $20(25.3)$ \\
$3-4$ & $22(27.8)$ \\
$5-6$ & $8(10.2)$ \\
$7-8$ & $6(7.6)$ \\
$\geq 9$ & $23(29.1)$ \\
Total & $79^{*}$ \\
Median $(\mathrm{IQR})=4(2-10)$, Range $; 1-35$ & \\
Highest spO $\mathbf{2}(\mathbf{\%})$ on admission & \\
$\leq 89$ & $21(30.4)$ \\
$90-94$ & $19(27.6)$ \\
$\geq 95$ & $29(42.0)$ \\
Total & $69^{*}$ \\
Mean $\pm \mathrm{SD}=89 \pm 10$, Range $; 54-99$ & \\
Lowest $\mathbf{S O}_{2}(\%)$ on admission & \\
$\leq 89$ & $74(96.1)$ \\
$90-94$ & $3(3.9)$ \\
$\geq 95$ & $0(0.0)$ \\
Total & $77^{*}$ \\
Mean $\pm \mathrm{SD}=65 \pm 16$, Range $; 30-91$ & \\
\hline
\end{tabular}

*Missing values not include

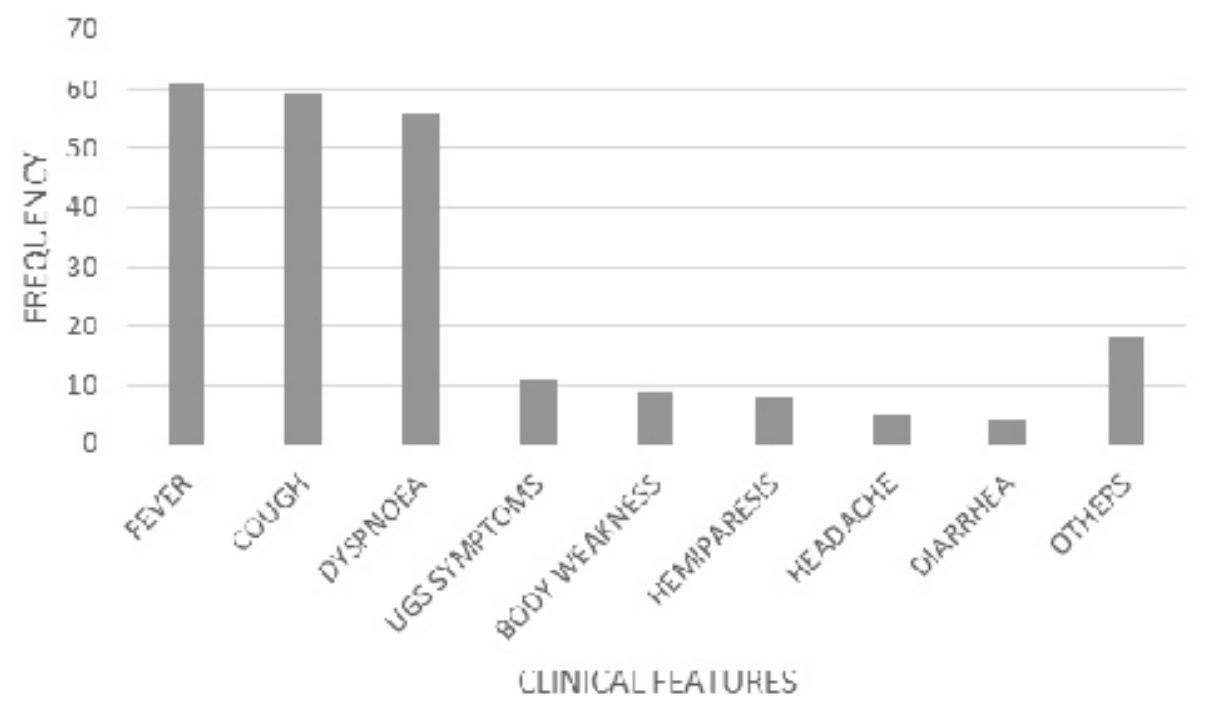

Figure 1: Distribution of Clinical features at presentation

Others: anorexia, loss of taste, confusion, irrational talk, vomiting and leg swelling UGS-Urogenital system 
Gomerep SS, McHenry SI, Kumbak FD, Galam NZ, Ogwuche JE, Bartekwa JW, Bako IB, Shehu NY, Isa SE.

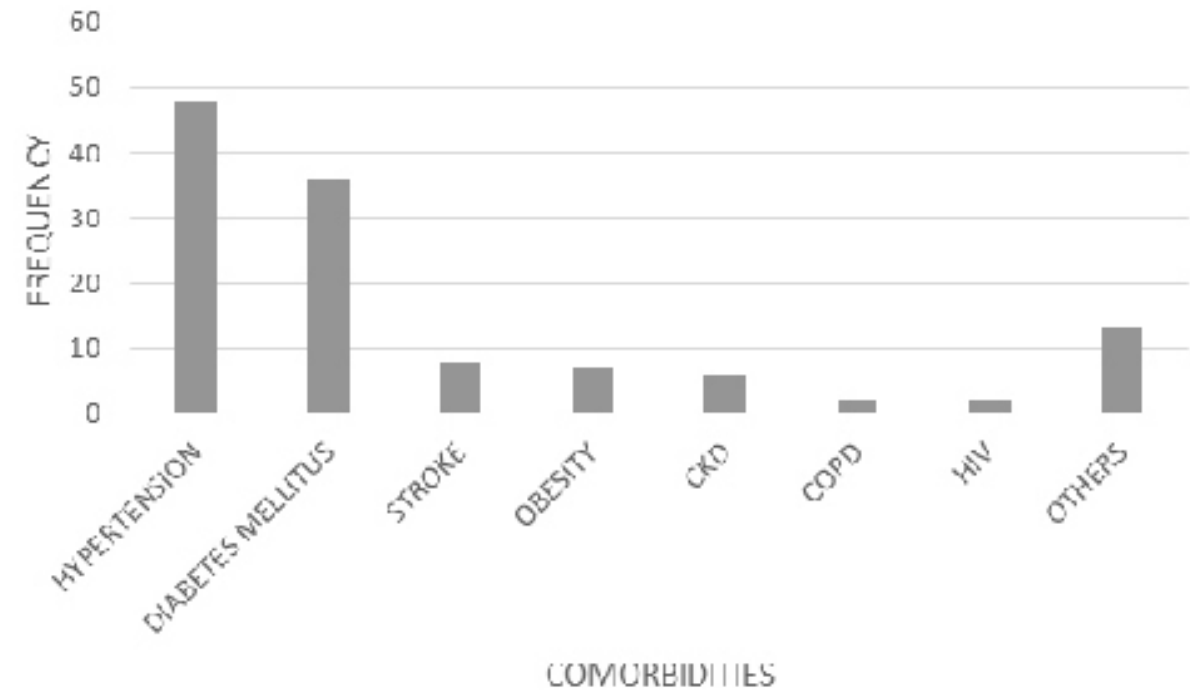

Figure 2: Comorbidities among the study population OTHERS: Prostrate cancer, liver cirrhosis, cardiomyopathy, psychosis, spinal deformity, benign prostatic hypertrophy, hemorrhoids, and hip dislocation.

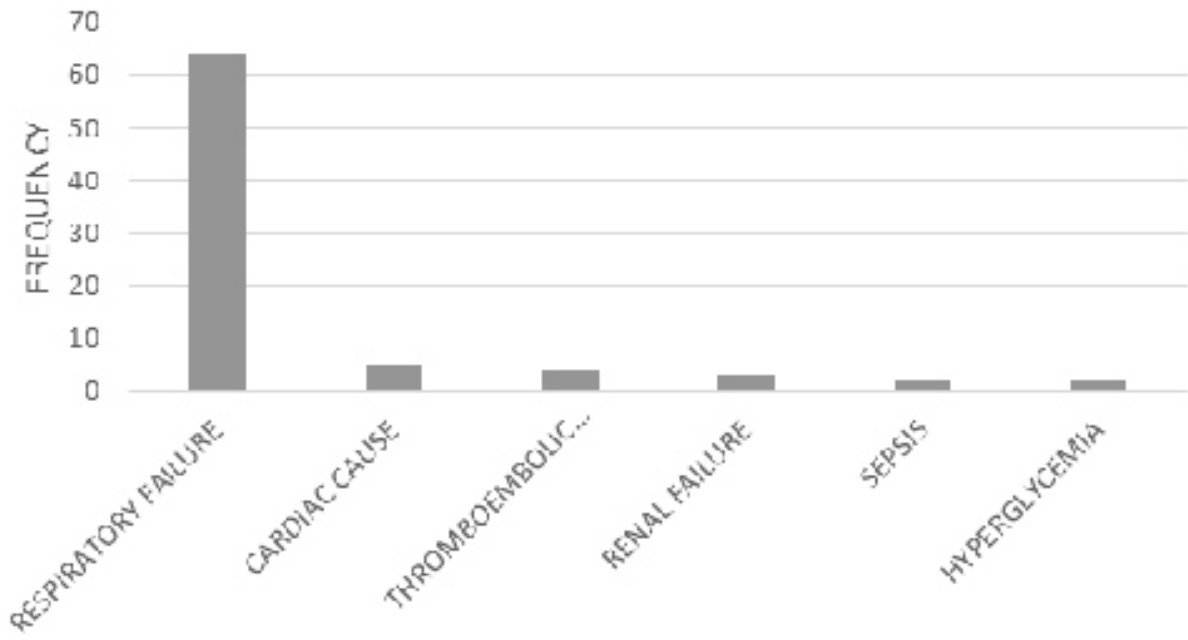

CAUSE OH DEAIH

Figure 3: Distribution of causes of death

Cardiac cause: cardiac arrest, cardiac failure and cardiogenic shock 


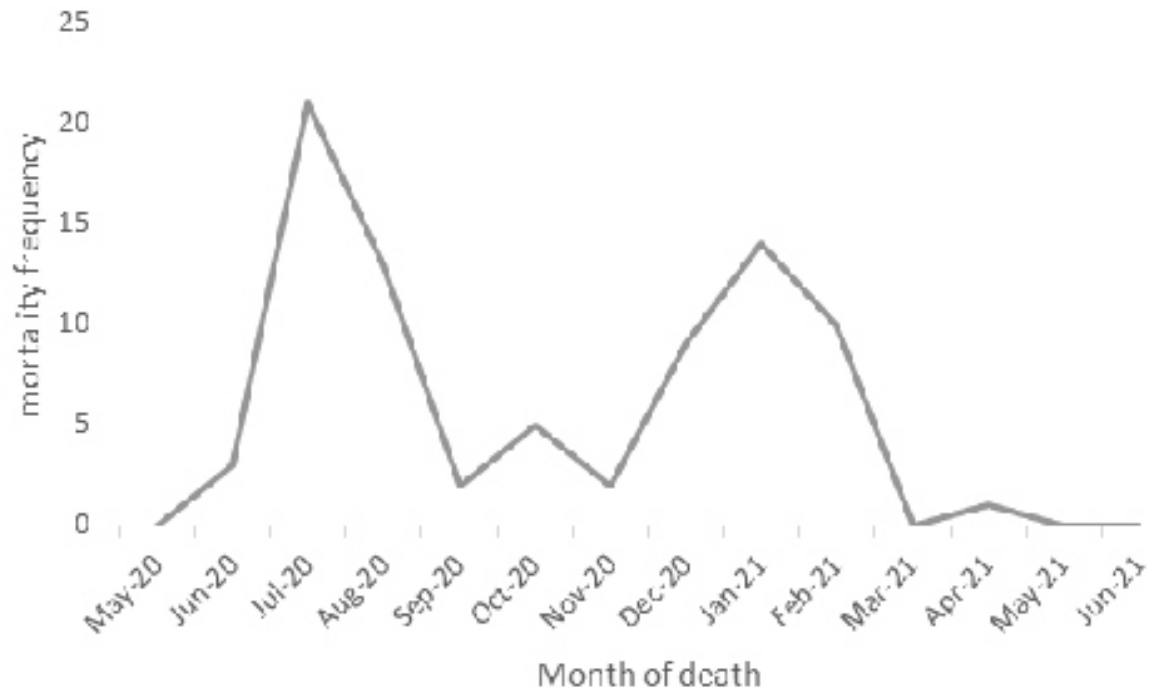

Figure 4: Mortality trend among COVID-19 in-patients

Table 3: Association between sex, co-morbidities and ventilator support among the patients

\begin{tabular}{llll}
\hline Characteristics & $\begin{array}{l}\text { Ventilator support } \\
\text { Not used } \\
\text { Freq. (\%) }\end{array}$ & $\begin{array}{l}\text { Used } \\
\text { Freq. (\%) }\end{array}$ & p-value \\
\hline Sex & $39(75.0)$ & $22(78.6)$ & 0.720 \\
Male & $13(25.0)$ & $6(21.4)$ & \\
Female & 52 & 28 & \\
Total & & & \\
Hypertension status & $26(50.0)$ & $22(78.6)$ & $\mathbf{0 . 0 1 3}$ \\
Hypertensive & $26(50.0)$ & $6(21.4)$ & \\
Non-Hypertensive & 52 & 28 & \\
Total & & $15(53.6)$ & 0.258 \\
Diabetes mellitus status & $21(40.4)$ & $13(46.4)$ & \\
Diabetic & $31(59.6)$ & 28 & \\
Non-Diabetic & 52 & & \\
Total & & $0(0.0)$ & $\mathbf{0 . 0 0 1 *}$ \\
Number of & & $7(25.0)$ & \\
comorbidities & $15(32.7)$ & $15(53.6)$ & \\
None & $8(15.4)$ & $6(21.4)$ & \\
Single & $17(32.7)$ & 28 & \\
Double & $10(19.2)$ & \\
Multiple & 52 & \\
Total &
\end{tabular}

* Likelihood ratio chi-square 


\section{Discussion}

Males, older age groups and retired workers were the majority of cases in the study population. This finding is similar to those in other studies which have shown that in patients with confirmed COVID-19 admitted to intensive care units, high mortality rate has been associated with increasing age, male sex, history of smoking, hypertension and diabetes mellitus. ${ }^{5,6,7}$ In a nationwide populationbased cohort study in Denmark, advanced age, male sex, comorbidity, higher levels of systemic inflammation and cellturnover were independent factors for mortality. Age was the strongest predictor for death, and moderate to high level of comorbidity were associated with a nearly two-fold increase in mortality. ${ }^{16}$ In addition, age above 60 years and being male where shown to be significantly associated with mortality in another study. ${ }^{17}$

Cough, fever and dyspnea where the commonest presenting symptoms among mortalities in our study population, and a combination of the three symptoms occurred in close to half of the patients. This was also seen in a cohort of 1000 patients in a New York cohort ${ }^{18}$ As these constellation of symptoms constitute a severe case of COVID 19 at presentation, physicians attending to such patients must give all the necessary attention because of the associated high mortality. The median duration of hospital stay was comparatively shorter than that observed in New York. ${ }^{17}$ This shows that our patients died shortly after admission and this may be related to limited availability of critical care for patients in our resource limited setting. This is supported by the fact that only half ever achieved a normal $\mathrm{SpO} 2$ at any point during admission and most of them died of respiratory failure. There is an urgent need to increase access to critical care services; ventilators and personnel trained in the use and maintenance of equipment.

Most of our study patients had at least one comorbidity. In a study among consecutive patients admitted to a hospital in Detroit, it was shown that up to $94 \%$ had at least one comorbidity. ${ }^{19}$ This finding strongly suggests a strong association between COVID 19 severity and comorbidities. Among our study patients, hypertension followed by diabetes where the two most common comorbidities, as was also seen among hospitalized patients in Wuhan and New York. ${ }^{19,}{ }^{20}$ In two studies in Lagos, Nigeria, hypertension followed by diabetes were the commonest comorbidities and severe symptoms and mortality were significantly higher among hypertensives just as survival rates were significantly lowered by the presence of an additional comorbidity to $50 \%$ from $91 \%$ for those with hypertension alone, and to $50 \%$ from $98 \%$ for all other patients. ${ }^{14,21}$ This also supports our finding that hypertension and additional comorbidity were associated with ventilator use which can be a surrogate measure of disease severity.

In a meta-analysis, a moderate positive association was found between diabetes and hypertension on one hand and COVID-19 severity and mortality on the other. Diabetes mellitus produces a 2.3fold increase in the risk of severity and a 2.5 -fold increase in mortality associated with COVID- $19,{ }^{22}$ which was similar to another study which showed an increased risk of $2.75 .{ }^{23}$ Compromised innate immune system due to chronic hyperglycemia, pro-inflammatory state characterized by inappropriate and exaggerated cytokine response and underlying pro-thrombotic hypercoagulable state have been implicated in this association ${ }^{24,25}$. In another 
meta-analysis, hypertension was shown to increase more than twice the risk of mortality and COVID-19 severity ${ }^{26 .}$ The association between hypertension and worse outcomes of COVID-19 infection may be due to the increased occurrence of comorbidities with advancing age, and also these comorbidities have a common complex pathological process associated with oxidative stress, inflammation and a prothrombotic state which results in vascular damage and vascular remodeling that are seen in individuals with type 2 diabetes, obesity and hypertension ${ }^{27,28}$

While the pattern of mortality look similar between developed and resource limited settings affecting the aged, with multiple comorbidities, the variation in the rate of mortality may be attributable to a much younger population in resource limited settings. The mortality trend showed a double peak corresponding to the first wave and second wave of infection in Nigeria but the higher mortality occurred during the first wave. This was the period of steep learning, shortages of equipment and laboratory support. Nigeria and the majority of African countries lack specialized medical capacity that is critical for handling severe cases of COVID-19 ${ }^{29}$, ${ }^{30}$. This further reinforces the need for developing capacity in emergency preparedness.

This study never the less, has been able to describe the clinical features of this novel disease in patients who died in our environment despite the absence of some important support such as laboratory and radiological investigation. A prospective study can build on this to identify risk factors for transmission, detailed clinical course of illness, risk factors for mortality and its treatment outcomes.

\section{Conclusion}

Among the cases of COVID 19 mortality in our centre, fever, cough and dyspnea were common at presentation as well as old age, male sex, hypertension and diabetes. Special attention must be given to the management of these comorbidities to improve the outcome of COVID-19 management.

\section{Reference}

1. Jee Y. World Health Organization. International Health Regulations Emergency Committee for the COVID-19 outbreak. Epidemiol Health. 2020;42:e2020013. doi: 10.4178/epih.e2020013. Epub 2020 Mar 19. PMID: 32192278; PMCID: PMC7285442.

2. Lwin MO, Lu J, Sheldenkar A et al. Global sentiments surrounding the COVID-19 pandemic on Twitter: analysis of Twitter trends. JMIR public health and surveillance, 2020. 6(2): e19447.

3. World Health Organization. Coronavirus Disease (COVID 19) Pandemic: Numbers at a glance. https://www.who.int/emergencies/di seases/novel-coronavirus-2019 accessed June 26, 2021

4. Lawal Y. Africa's low COVID-19 mortality rate: A paradox? International Journal of Infectious Diseases 2021; 102:118-122.

5. Bhatraju PK, Ghassemieh BJ, Nichols M et al. Covid-19 in critically ill patients in the Seattle Region - case series. N Engl J Med. 2020;382: $\begin{array}{llllllllll}2 & 0 & 1 & 2 & - & 2 & 0 & 2 & 2\end{array}$. https://doi.org/10.1056/NEJMoa200 4500.

6. Zhou F, Yu T, Du R et al. Clinical course and risk factors for mortality of adult inpatients with COVID-19 in Wuhan, China: a retrospective cohort $\mathrm{st} u \mathrm{~d} y$. L a n c e t 2020 ; 395 (10229): $1054-1062$. https://doi.org/10.1016/S01406736(20)30566-3. 
Gomerep SS, McHenry SI, Kumbak FD, Galam NZ, Ogwuche JE, Bartekwa JW, Bako IB, Shehu NY, Isa SE.

7. Ioannou GN, Locke E, Green P et al. Risk Factors for Hospitalization, Mechanical Ventilation, or Death Among 10131 US Veterans With SARS-CoV-2 Infection. JAMA Netw Open 2020 Sep ;3 (9):e2022310. doi: 10.1001/jamanetworkopen.2020.223 10. PMID: 32965502; PMCID: PMC7512055.

8. Caramelo F, Ferreira N, Oliveiros B. Estimation of risk factors for COVID19 mortality-preliminary results. $\mathrm{M}$ e d R x i v 2020 . d o i : https://doi.org/10.1101/2020.02.24.2 0027268

9. Boulle A, Davies MA, Hussey $\mathrm{H}$ et al. Risk factors for COVID-19 death in a population cohort study from the Western Cape Province, South Africa. Clin Infect Dis 2020 [cited 2021 4/06/2021]; Available from: https://www.ncbi.nlm.nih.gov/pmc/a rticles/PMC7499501/.

10. Okonji EF, Okonji OC, Mukumbang FC et al. Understanding varying COVID-19 mortality rates reported in Africa compared to Europe, Americas and Asia. Tropical Medicine \& International Health 2021;26(7):716719.

11. Paintsil E. COVID-19 threatens health systems in sub-Saharan Africa: the eye of the crocodile. The journal of clinical investigation $2020 ; 130(6): 2741-2744$. https://doi.org/10.1172/JCI138493.

12. Nigerian Centre for Disease Control. COVID-19 in Nigeria. Available at https://covid19.ncdc.gov.ng/ Accessed June 262021.

13. Hassan Z, Hashim MJ, Khan G. Population risk factors for COVID-19 deaths in Nigeria at sub-national level. The Pan African Medical Journal 2020. 35(Suppl 2): 131. $\mathrm{Pub} 1 \mathrm{ish}$ ed 2020 A u g 4 . doi:10.11604/pamj.supp.2020.35.13

\subsection{8}

14. Osibogun A, Abayomi A, KanmaOkafor $\mathrm{O}$ et al. Morbidity and mortality outcomes of COVID-19 patients with and without hypertension in Lagos, Nigeria: A retrospective cohort study. Global Health Research and Policy Under review DOI: 10.21203/rs.3.rs70014/v3

15. Stata Corporation, College Station, $\mathrm{T}$ e $\mathrm{x}$ a s, U S A . A t https://www.stata.com

16. Holler JG, Eriksson R, Jensen TØ et al. First wave of COVID-19 hospital admissions in Denmark: a nationwide population-based cohort study. BMC I $\mathrm{n} \mathrm{fect} \mathrm{D} \mathrm{is} 2021 ; 21$ : 39.https://doi.org/10.1186/s12879020-05717-w

17. Suleyman G, Fadel RA, Malette KM, et al. Clinical Characteristics and Morbidity Associated With Coronavirus Disease 2019 in a Series of Patients in Metropolitan Detroit. J A M A N e t w O p e n. 2020 ; 3 (6): e 2012270 . doi : 10.1001/jamanetworkopen.2020.122 70. PMID: 32543702; PMCID: PMC7298606.

18. Argenziano MG, Bruce SL, Slater CL et al. Characterization and clinical course of 1000 patients with coronavirus disease 2019 in New York: retrospective case series. BMJ 2020 ; 369 :m 1996. doi: $10.1136 / \mathrm{bmj} . \mathrm{m} 1996$. PMID: 32471884; PMCID: PMC7256651.

19. Richardson S, Hirsch JS, Narasimhan $\mathrm{M}$ et al. Presenting Characteristics, Comorbidities, and Outcomes Among 5700 Patients Hospitalized With COVID-19 in the New York City Area [published correction appears in JAMA. 2020 May 26;323(20):2098]. JAMA. $2020 ; 323(20): 2052-2059$. 
doi:10.1001/jama.2020.6775

20. Zhou F, Yu T, Du R et al. Clinical course and risk factors for mortality of adult inpatients with COVID-19 in Wuhan, China: a retrospective cohort $\mathrm{s} \mathrm{t} \mathrm{u} \mathrm{d} \mathrm{y.} \mathrm{L} \mathrm{a} \mathrm{n} \mathrm{c} \mathrm{e} \mathrm{t.}$ 2020;395(10229):1054-1062. doi: 10.1016/S0140-6736(20)30566-3. Epub 2020 Mar 11. Erratum in: $\mathrm{L}$ a $\mathrm{n} \mathrm{c}$ e t . $2020 \quad \mathrm{M}$ a r 28;395(10229):1038. Erratum in: $\mathrm{L}$ a n c e t. $2020 \quad \mathrm{M}$ a r 28;395(10229):1038. PMID: 32171076 ; PMCID: PMC7270627.

21. Osibogun A, Balogun M, Abayomi A et al. Outcomes of COVID-19 patients with comorbidities in southwest Nigeria. PLoS ONE 2021; 16 ( 3 ): $\quad$ e 0248281 . https://doi.org/10.1371/journal.pone. 0248281

22. de Almeida-Pititto B, Dualib PM, Zajdenverg L et al. Severity and mortality of COVID 19 in patients with diabetes, hypertension and cardiovascular disease: a metaanalysis. Diabetol Metab Syndr 2020; 12 : 75 . https://doi.org/10.1186/s13098-02000586-4

23. Kumar A, Arora A, Sharma $\mathrm{P}$ et al. Is diabetes mellitus associated with mortality and severity of COVID-19? A meta-analysis. Diabetes Metab Syndr $2020 ; 14(4): 535-45$. https://doi.org/10.1016/j.dsx.2020.04 .044 .

24. Chan JW, Ng CK, Chan $\mathrm{YH}$ et al. Short term outcome and risk factors for adverse clinical outcomes in adults with severe acute respiratory syndrome (SARS). Thorax $2003 ; 58(8)$ : $686-9$. https://doi.org/10.1136/thorax.58.8.6 86.

25. Morra ME, Van Thanh L, Kamel MG et al. Clinical outcomes of current medical approaches for Middle East respiratory syndrome: A systematic review and meta-analysis. Rev Med Virol $2018 ; 28$ (3): e 1977. https://doi.org/10.1002/rmv.1977.

26. Pranata R, Lim MA, Huang I et al. Hypertension is associated with increased mortality and severity of disease in COVID-19 pneumonia: A systematic review, meta-analysis and meta-regression. J Renin Angiotensin A $1 \mathrm{~d}$ o s t e r o n e $\mathrm{S}$ y s t 2020;21(2):1470320320926899. https://doi.org/10.1177/1470320320 926899.

27. Petrie JR, Guzik TJ, Touyz RM. Diabetes, hypertension, and cardiovascular disease: clinical insights and vascular mechanisms. Can J Cardiol 2018;34(5):575-84. https://doi.org/10.1016/j.cjca.2017.1 2.005 .

28. Iccarino $\mathrm{G}$, Grassi G, Borghi $\mathrm{C}$ et al. Age and Multimorbidity Predict Death Among COVID-19 Patients. Results of the SARS-RAS Study of the Italian Society of Hypertension. Hypertension 2020;76:366-72. https://doi.org/10.1161/HYPERTEN SIONAHA.120.15324Hypertension.

29. Murthy S, Leligdowicz A, Adhikari NKJ: Intensive care unit capacity in low-income countries: A systematic $\mathrm{r}$ e vi e w. $P L O S O n e$ 2015 ; 10 ( 1 ) : e 0116949 . 10.1371/journal.pone.0116949

30. The times of Africa: Africa: Shortage of Not Only Ventilators! Published A p ri $\quad 20, \quad 2020$ https://thetimesofafrica.com/africasho 
